\title{
Valproic acid exhibits anti-tumor activity selectively against EGFR/ErbB2/ErbB3- coexpressing pancreatic cancer via induction of ErbB family members- targeting microRNAs
}

Tingting Lin ${ }^{1,2,3 \dagger}$, Qun Ren ${ }^{1,2 \dagger}$, Weimin Zuo ${ }^{2,4 \dagger}$, Ruxue Jia ${ }^{2}$, Linhui Xie ${ }^{5}$, Rong Lin², Hu Zhao ${ }^{1,2}$, Jin Chen², Yan Lei ${ }^{2}$, Ping Wang ${ }^{2}$, Huiyue Dong ${ }^{2}$, Lianghu Huang ${ }^{2}$, Jinquan Cai ${ }^{1}$, Yonghai Peng ${ }^{6}$, Zongyang Y ${ }^{6}$, Jianming Tan ${ }^{1,2}$ and Shuiliang Wang ${ }^{1,2^{*}}$

\begin{abstract}
Background: Deregulated ErbB signaling plays an important role in tumorigenesis of pancreatic cancer. However, patients with pancreatic cancer benefit little from current existed therapies targeting the ErbB signaling. Here, we explore the potential anti-tumor activity of Valproic acid against pancreatic cancer via targeting ErbB family members.
\end{abstract}

Methods: Cell viability assay and apoptosis evaluation were carried out to determine the efficacy of VPA on pancreatic cancer cells. Western blot analyses were performed to determine the expression and activation of proteins. Apoptosis enzyme-linked immunosorbent assay was used to quantify cytoplasmic histone associated DNA fragments. Lentiviral expression system was used to introduce overexpression of exogeneous genes or gene-targeting short hairpin RNAs (shRNAs). qRT-PCR was carried out to analyze the mRNAs and miRNAs expression levels. Tumor xenograft model was established to evaluate the in vivo anti-pancreatic cancer activity of VPA.

Results: VPA preferentially inhibited cell proliferation/survival of, and induced apoptosis in EGFR/ErbB2/ErbB3coexpressing pancreatic cancer cells within its clinically achievable range $[40 \sim 100 \mathrm{mg} / \mathrm{L}(0.24 \sim 0.6 \mathrm{mmol} / \mathrm{L})]$. Mechanistic investigations revealed that VPA treatment resulted in simultaneous significant down-regulation of EGFR, ErbB2, and ErbB3 in pancreatic cancer cells likely via induction of ErbB family members-targeting microRNAs. Moreover, the antipancreatic cancer activity of VPA was further validated in tumor xenograft model.

Conclusions: Our data strongly suggest that VPA may be added to the treatment regimens for pancreatic cancer patients with co-overexpression of the ErbB family members.

Keywords: Valproic acid, Pancreatic cancer, ErbB family members, microRNAs

\footnotetext{
* Correspondence: shuiliang.wang@xmu.edu.cn

†Tingting Lin, Qun Ren and Weimin Zuo contributed equally to this work.

'Department of Urology, The 900th Hospital of the Joint Logistics Team (the

Former Fuzhou General Hospital), Fujian Medical University, Fuzhou 350025,

China. 156 Xi'er Huan Bei Road, Fuzhou 350025, Fujian Province, China

${ }^{2}$ Fujian Key Laboratory of Transplant Biology, Affiliated Dongfang Hospital,

Xiamen University School of Medicine, Fuzhou 350025, Fujian Province,

China

Full list of author information is available at the end of the article
}

(c) The Author(s). 2019 Open Access This article is distributed under the terms of the Creative Commons Attribution 4.0 International License (http://creativecommons.org/licenses/by/4.0/), which permits unrestricted use, distribution, and reproduction in any medium, provided you give appropriate credit to the original author(s) and the source, provide a link to the Creative Commons license, and indicate if changes were made. The Creative Commons Public Domain Dedication waiver (http://creativecommons.org/publicdomain/zero/1.0/) applies to the data made available in this article, unless otherwise stated. 


\section{Background}

The worldwide mortality of pancreatic cancer ranks eighth and ninth among all cancers in male and female, respectively [1]. Owing to lack of clinically validated screening methods for pancreatic cancer in the curative stage, fewer than $20 \%$ of patients will be diagnosed with localized tumors and surgery remains the only option for cure [2]. Thus far the overall 5-year survival rate among patients with pancreatic cancer is $<5 \%[3,4]$.

Mainly arising from pancreatic ductal cells, pancreatic cancer could also develop from acinar cells as evidenced [5-7]. Extensively genetic studies have revealed that during the progression of three broad stages of pancreatic cancer, acquired somatic mutations in oncogenes and tumor suppressor genes accumulate and account for initiation and aggressive development of this malignant disease. These mutations occur most frequently in KRAS, CDKN2A, TP53 and SMAD4 [8-10]. Activating mutations of the KRAS oncogene, which encodes a member of the RAS family of GTP-binding proteins, are the most common genetic abnormality presenting in approximately $95 \%$ of pancreatic tumors analyzed [11, 12]. In addition, wild-type KRAS is also normally activated in response to the binding of extracellular signals such as growth factors to receptor tyrosine kinases (RTKs) [13]. Among all subfamilies of RTKs, the ErbB family members consisting of the epidermal growth factor receptor EGFR (ErbB1), HER2 (ErbB2), HER3 (ErbB3), and HER4 (ErbB4) play important role in the initiation and maintenance of a variety of human cancers, including pancreatic cancer $[14,15]$. Accumulated evidence shows that the ErbB receptors overexpress in approximately $60 \%$ of pancreatic cancers [16]. Collectively, deregulated RTKs/ RAS/RAF/MEK/MAPK signaling pathway is undoubtedly important for pancreatic cancer biology, and extensive efforts have been taken to target this pathway for systemic therapy [17-20].

In addition to gene amplification and mutation, alterations in chromatin structure by histone modification and/or DNA methylation also play a vital role in transcriptional regulation of oncogene or tumor suppressors in human cancers [21]. Thus, epigenetic targeting is emerging as a promising therapeutic strategy for cancer treatment. Histone deacetylases (HDACs), whose deregulation is evidenced to play an important role in aberrant gene expression in tumorigenesis, have long been recognized as druggable targets [22]. We have previously found that the class I HDAC inhibitor (HDACi), entinostat (also known as MS-275 or SNDX-275) specifically enhanced expression of miR-125a, miR-125b, and miR-205, which acted in concert to downregulate ErbB2 and ErbB3 in ErbB2-overexpressing breast cancer cells $[23,24]$. In our attempt to identify novel strategy targeting RTKs signaling in pancreatic cancer, we noticed that
Valproic acid (VPA), a safely used anti-convulsant drug in the treatment of epilepsy and other seizure disorders, was reported to exert potent anti-tumor activity in a number of cancers owing to its HDACi capability [25]. However, the underlying mechanism of VPA against human cancers remains poorly understood. In our current study, we have explored the potential therapeutic efficacy of VPA on pancreatic cancer using both an in vitro cell culture system and an in vivo tumor xenograft model. The molecular basis of VPA-mediated anti- pancreatic cancer activity was also elucidated.

\section{Methods}

\section{Reagents and antibodies}

Valproic acid and LY294002 were purchased from Sigma-Aldrich (St. Louis, MO, USA) and dissolved in $\mathrm{ddH}_{2} \mathrm{O}$ or dimethyl sulfoxide (DMSO) to make a stock solution at $500 \mathrm{mmol} / \mathrm{L}$ or $20 \mathrm{mmol} / \mathrm{L}$, respectively. All the stock solutions were stored at $-20^{\circ} \mathrm{C}$. Recombinant human NRG-1 protein ab50227 was product from abcam (Cambridge, MA, USA).

MISSION $^{\circ}$ Non-target shRNA, which does not target human and mouse genes, control vector (pLKO.1-ConshRNA), and pLKO.1 containing human erbB3 shRNA (pLKO.1-ErbB3shRNA) were purchased from Sigma. The packaging plasmids psPAX2 and pMD2.G for lentiviral expression vector were from Addgene Inc. (Cambridge, MA, USA).

Antibodies were obtained as follows: EGFR, ErbB2, ErbB3, PARP, Cleaved Caspase-3 (Asp175) (5A1E), P-MAPK (E10), MAPK, P-Akt (Ser473), Akt, STAT3, P-STAT3 (Tyr705), p21, Cyclin D1, RAS, Ki67 (Cell Signaling Technology, Inc., Beverly, MA, USA); $\beta$-actin (AC-75) (Sigma). All other reagents were purchased from Sigma unless otherwise specified.

\section{Cells and cell culture}

Human pancreatic adenocarcinoma cell lines HPAF-II, MPanc96, MiaPaca-2, and Panc-1 were purchased from ATCC (Manassas, VA, USA) and maintained in RPMI1640 medium supplemented with $10 \%$ fetal bovine serum (FBS). HEK293T human embryonic kidney cells were maintained in DMEM/F12 medium containing $10 \%$ FBS. All cell lines were cultured in a $37^{\circ} \mathrm{C}$ humidified atmosphere containing $95 \%$ air and $5 \% \mathrm{CO}_{2}$ and were split twice a week.

\section{Cell viability assay}

The CellTiter96AQ cell proliferation kit (Promega, WI, USA) was used to determine cell viability as we previously described [26]. For cell staining assays, human pancreatic cancer cells HPAF-II, MPanc96, MiaPaca-2, and Panc-1 were plated onto 24-well plates and incubated at $37^{\circ} \mathrm{C}$ with $5 \% \mathrm{CO} 2$. After $24 \mathrm{~h}$, the culture 
medium was replaced with $700 \mu \mathrm{l}$ of medium containing $0.5 \% \mathrm{FBS}$ or the same medium containing indicated concentrations of VPA. Cells were incubated in a $37^{\circ} \mathrm{C}$ humidified atmosphere containing $95 \%$ air and $5 \% \mathrm{CO}_{2}$ for $72 \mathrm{~h}$. The percentages of surviving cells from each group relative to controls, defined as $100 \%$ survival, was determined by reduction of MTS following by staining with $0.5 \%$ crystal violet for visualization of viable cells.

\section{Western blotting analysis and quantification of apoptosis}

Protein expression and activation were determined by western blotting analysis as previously described [27]. In brief, equal amounts of cell lysates in a buffer were boiled in sodium dodecyl sulfate sample buffer, resolved by sodium dodecyl sulfate polyacrylamide gel electrophoresis and western blotted with specific antibodies directed against EGFR, ErbB2, and ErbB3, PARP, Cleaved Caspase-3 (C-Casp-3), P-Akt, Akt, P-MAPK, MAPK, P-STAT3, STAT3, RAS, or $\beta$-actin, as described in the figure legends. For quantification of apoptosis, an apoptosis enzyme-linked immunosorbent assay kit (Roche Diagnostics Corp., Indianapolis, IN, USA) was used to quantitatively measure cytoplasmic histone associated DNA fragments (mononucleosomes and oligonucleosomes) as previously reported [27]. In addition, cell apoptosis was also detected with flow cytometry assay. Briefly, cells upon treatment with indicated concentration of VPA for $24 \mathrm{~h}$ or $48 \mathrm{~h}$ were harvested and co-stained with propidiumiodide (PI) and annexin V-fluorescein isothiocyanate (annexin V-FITC). The cells were finally analyzed with a FACSort flow cytometer (Becton-Dickinson, Franklin, NJ, USA). Data were acquired after analysis of at least 10,000 events.

\section{Construction of lentiviral expression vector pLEX-erbB2 and production of lentivirus}

The coding sequence of human $E r b B 2$ was amplified from pDsRed-erbB2 by PCR with the following forward primer: 5' -ATAGCGGCCGCATGGAGCTGGCGGCCTTGT-3' and reverse primer: 5'-GCGACGCGTTCACACTGGC ACGTCCAGACC-3'. The amplified fragments were inserted into the lentiviral vector pLEX-MCS (Open Biosystem, Huntsville, AL, USA). After sequencing verification, the recombinant was nominated as pLEX-erbB2. Lentiviral production was performed as described [28].

\section{Analysis of mRNA and miRNA expression with real-time quantitative reverse transcriptase PCR (qRT-PCR)}

Total RNA was prepared using TRIZOL reagent (Invitrogen, Carlsbad, CA, USA). First-strand cDNA was generated using High-Capacity cDNA Reverse Transcription Kit (Applied Biosystems, Carlsbad, CA, USA) following the manufacturer's instructions. The analysis of human EGFR, ErbB2 and ErbB3 mRNA expression was examined by conventional RT-PCR as we had described previously [24]. To quantify the mRNA levels, qRT-PCR was performed using the FastStart Universal SYBR Green Master Mixes (Roche) by a 7900HT Fast Real-Time PCR system (Applied Biosystems). The expression of $\beta$-actin was used as an internal control for both conventional RT-PCR and qRT-PCR. The relative mRNA levels were calculated using the comparative $\mathrm{Ct}$ method $(\Delta \Delta \mathrm{Ct})$. Sequences of specific primers used are listed in Additional file 1: Table S1.

The expression of mature miR-133a, miR-133b, miR-125a, miR-125b, miR-205, and RNU6B was measured by qRT-PCR using TaqMan Assays (Applied Biosystems) as described previously [24]. The relative miRNA levels were calculated using the comparative $\mathrm{Ct}$ method $(\Delta \Delta \mathrm{Ct})$.

\section{Tumor xenograft model}

Athymic nu/nu mice (Shanghai SLAC Laboratory Animal Co. Ltd., Shanghai, China) were maintained in accordance with the Institutional Animal Care and Use Committee (IACUC) procedures and guidelines approved by the Affiliated Dongfang Hospital of Xiamen University School of Medicine IACUC. Five $\times 10^{6}$ HPAF-II or MPanc96 cells were suspended in $50 \mu \mathrm{L}$ of PBS, mixed with Matrigel (BD Biosciences) and injected subcutaneously into the flanks of 5-week-old female mice. Tumor formation was assessed by palpation and measured with fine calipers three times a week. Tumor volume was calculated by the formula: Volume $=($ Length $\times$ Width $\left.^{2}\right) / 2$, which was statistically analyzed as we described previously [28]. When tumors reach $\sim 150 \mathrm{~mm}^{3}$, mice were randomly assigned into two groups $(n=3): 1)$ control mice received intraperitoneal (i.p.) injection of $100 \mu \mathrm{l}$ of PBS; 2) mice received i.p. injection of VPA $(500 \mathrm{mg} / \mathrm{kg})$ in $100 \mu \mathrm{l}$ PBS daily for consecutive 12 days. At the end of study, mice were euthanized according to approved protocol. Tumors were excised and subjected to immunohistochemistry analyses and qRT-PCR measurement of miRNAs expression.

\section{Immunohistochemistry (IHC)}

IHC was performed as described previously [28]. The specificity of all antibodies - EGFR (Cell Signaling Technology; rabbit polyclonal; cat\#2232; dilution 1:100 in TBST + 1\% BSA $w / v$ ), ErbB2 (EMD Chemicals; mouse monoclonal 96G; cat\#OP14T; dilution 1:500 in TBST + 1\% BSA w/v), ErbB3 (Spring Bioscience, Pleasanton, CA, USA; rabbit monoclonal SP71; cat\# M3710; dilution 1:200 in TBST $+1 \%$ BSA w/v), Ki-67 (rabbit monoclonal SP6; dilution 1:500 in TBST + 1\% BSA), cleaved caspase-3 (rabbit polyclonal; 1:1000) - has been confirmed by both positive and negative controls. Antibody complexes were visualized with IP Flex DAB (Biocare). All sections were counterstained in Mayer's hematoxylin, 
nuclei blued in $1 \%$ ammonium hydroxide, cleared in $\mathrm{xy}$ lene and cover glass mounted by synthetic resin.

\section{Statistical analysis}

All results were confirmed by at least three independent experiments. Data are presented as mean \pm SD. "Tow" sided Student's $t$-tests were used for comparisons of means of quantitative data between groups. A $P$-value $<0.05$ was deemed statistically significant. All statistical analyses were conducted with the software StatView v5.1 from SAS Institute Inc. (Cary, NC).

\section{Results}

VPA preferentially inhibits cell proliferation/survival of EGFR/ErbB2/ErbB3-coexpressing pancreatic cancer cells in vitro

To explore whether VPA might show any therapeutic effect on pancreatic cancer, we investigated its anti-proliferative/ anti-survival activities in the EGFR/ErbB2/ErbB3-coexpressing (HPAF-II, MPanc96) and ErbB3-negative (MiaPaca-2, Panc-1) pancreatic cancer cell lines (Fig. 1a). We found that VPA inhibited proliferation/survival of all cells in a dose-dependent manner. The EGFR/ErbB2/ErbB3-coexpressing cells were much more sensitive than ErbB3-negative cells to VPA-induced growth inhibition (Fig. 1b). The $\mathrm{IC}_{50}$ values were $0.856 \mathrm{mmol} / \mathrm{L}$ and $0.961 \mathrm{mmol} / \mathrm{L}$ for HPAF-II and MPanc96 cells, and $2.428 \mathrm{mmol} / \mathrm{L}$ and 3.709 $\mathrm{mmol} / \mathrm{L}$ for MiaPaca-2 and Panc-1 cells, respectively. The inhibitory effect of VPA on proliferation/survival of pancreatic cancer cells was further supported by cell staining with crystal violet showing that the survived cells decreased significantly while treating with increased dose of VPA (Fig. 1c). In an effort to investigate the underlying mechanism, we found that while the expression of cyclin-dependent kinase (CDK) inhibitor P21, one of the most commonly induced genes by HDACi [29],

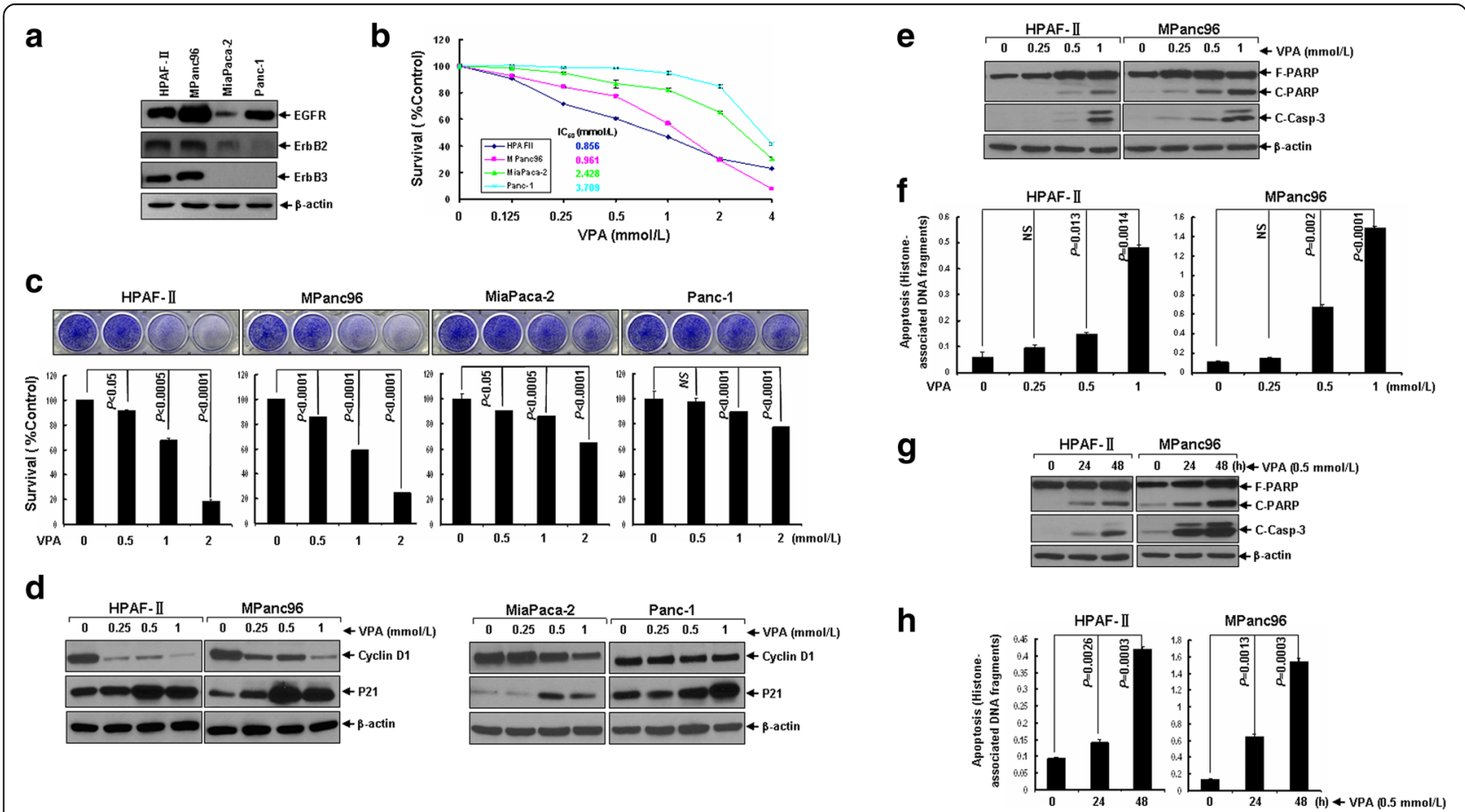

Fig. 1 VPA selectively inhibits proliferation/survival of EGFR/ErbB2/ErbB3-coexpressing pancreatic cancer cells. a Western blotting analysis of the expression of EGFR, ErbB2, and ErbB3 in pancreatic cancer cells. b Human pancreatic cancer cells were plated onto 96-well plates with complete culture medium (RPMI1640, 10\% FBS). After $24 \mathrm{~h}$, the medium was replaced with control medium (fresh RPMI1640, 0.5\% FBS) or same medium containing indicated concentrations of VPA for another $72 \mathrm{~h}$ incubation. The percentages of surviving cells from each treatment to controls, defined as 100\% survival, were determined by reduction of MTS. c Pancreatic cancer cells were grown in triplicates in the absence or presence of indicated concentrations of VPA for $72 \mathrm{~h}$. The percentages of surviving cells from each group relative to controls, defined as $100 \%$ survival, was determined by reduction of MTS following by staining with $0.5 \%$ crystal violet for visualization of viable cells. Bars, S.D. All data show the representative of three independent experiments. $\mathbf{d}$ HPAF-II and MPanc96 cells were treated with different concentrations of VPA as indicated for $24 \mathrm{~h}$. Cells were harvested and subjected to western blot analyses with specific antibodies directed against Cyclin D1, P21 or $\beta$-actin. e, f HPAF- $I$ and MPanc96 cells untreated or treated with different concentrations of VPA as indicated for $24 \mathrm{~h}$ were harvested and subjected to western blot analyses with specific antibodies directed against PARP, Cleaved Caspase-3 (C-Casp-3) or $\beta$-actin (e), or apoptotic ELISA (f). g, h HPAF-II and MPanc96 cells untreated or treated with VPA $(0.5 \mathrm{mmol} / \mathrm{L})$ for $24 \mathrm{~h}$ or $48 \mathrm{~h}$ were harvested and subjected to western blot analyses with specific antibodies directed against PARP, C-Casp-3 or $\beta$-actin (g), or apoptotic ELISA (h). Bars, S.D. All data show the representative of three independent experiments 
was significantly induced in all four pancreatic cancer cell lines upon treatment with VPA; however, the protein levels of cyclin D1 were only significantly decreased in the EGFR/ErbB2/ErbB3-coexpressing HPAF-II and MPanc96 cells (Fig. 1d).

In addition to growth inhibition, we then studied whether VPA might induce apoptosis in pancreatic cancer cells. Western blot analyses revealed that treatment with VPA induced PARP cleavage and activation of caspase-3 evidenced by the increase of cleaved caspase- 3 in the HPAF-II and MPanc96 cells dramatically (Fig. 1e and g), but only marginally promoted apoptosis in MiaPaca- 2 and Panc-1 (data not shown), suggesting that VPA selectively induced caspase-dependent apoptosis in the EGFR/ErbB2/ ErbB3-coexpressing pancreatic cancer cells. Furthermore, a quantitative apoptotic ELISA showed that the induction fold of apoptosis significantly increased with prolonged treatment or increased dose of VPA (Fig. If and h), suggesting that VPA induced apoptosis in the EGFR/ErbB2/ ErbB3-coexpressing pancreatic cancer cells in both doseand time-dependent manners. VPA-induced apoptosis in the EGFR/ErbB2/ErbB3-coexpressing pancreatic cancer cells was also confirmed by flow cytometry analysis (Additional file 2: Figure S1). Collectively, our results demonstrate that VPA selectively exerts anti-tumor activity in vitro through induction of growth inhibition as well as apoptosis in the EGFR/ErbB2/ErbB3-coexpressing pancreatic cancer cells.

\section{VPA treatment down-regulates expression of EGFR,}

\section{ErbB2, as well as ErbB3 in pancreatic cancer cells}

ErbB signaling exerts a broad spectrum of tumor biological functions which play important roles in several hallmarks of cancer [30]. We previously showed that SNDX-275, a class I-selective HDACi, induced apoptosis in ErbB2-overexpressing breast cancer cells via downregulation of both ErbB2 and ErbB3 [23]. In our attempt to determine the underlying mechanisms of anti-pancreatic cancer activity of VPA, the possible effect of VPA on expression of EGFR, ErbB2 and ErbB3 was investigated. As anticipated, upon treatment with VPA, the protein levels of EGFR, ErbB2 and ErbB3 in HPAF-II cells, and that of both EGFR and ErbB3 in MPanc96 cells were significantly reduced in a time-dependent manner (Fig. 2). Interestingly, while the expression of ErbB2 in MPanc96 cells decreased dramatically in a relative short time exposure to VPA, it recovered to some extent upon prolonged treatment with VPA (Fig. 2), which may reflect the happening of a feedback upregulation of ErbB2 in MPanc96 cells being similar to a phenomenon reported in breast cancer cells previously [31, 32]. Consequently, down-regulation of the ErbB family members resulted in significant inactivation of downstream Akt and MAPK signalings but not Stat3 signaling evidenced

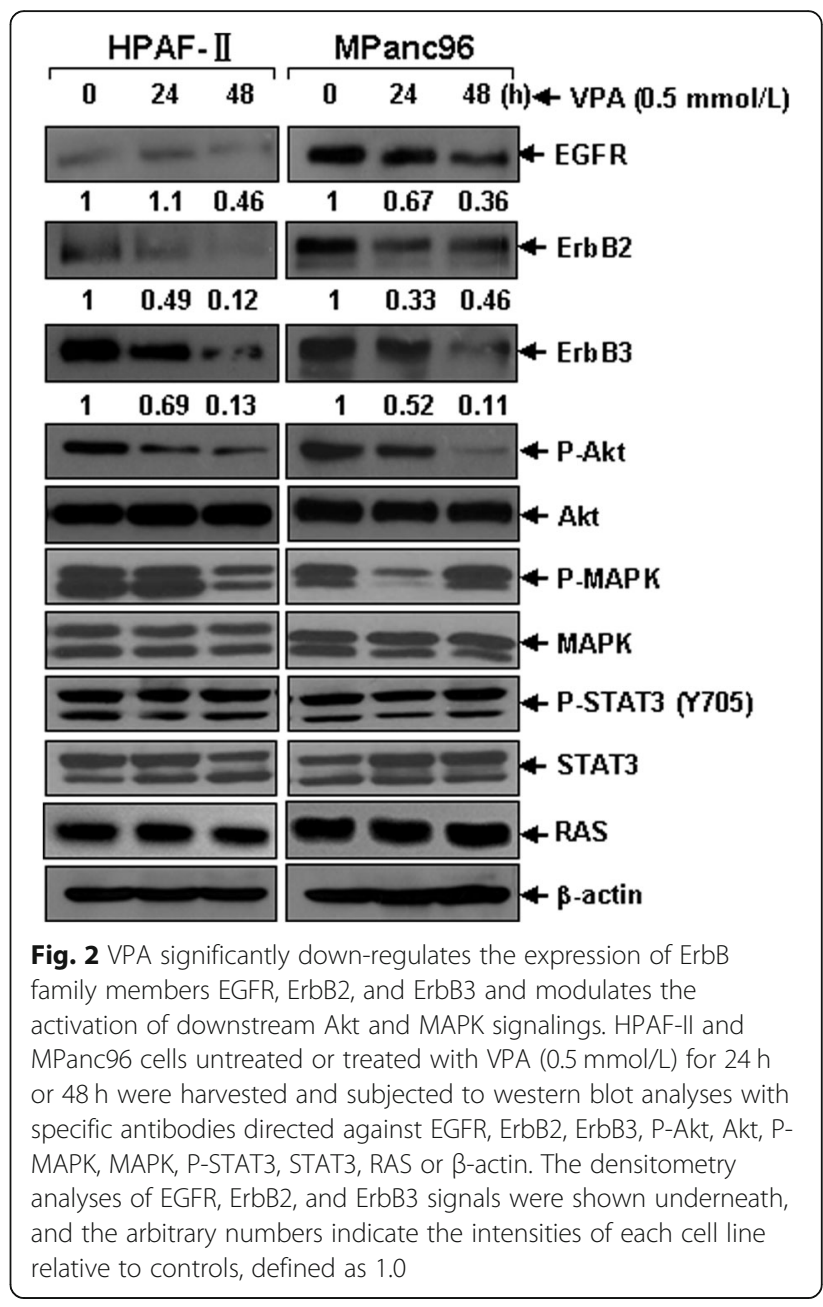

by decreased levels of both P-Akt and P-MAPK in HPAF-II and MPanc96 cells treated with VPA (Fig. 2). However, the expression of RAS remained unaltered. Thus, VPA treatment simultaneously down-regulates expression of EGFR, ErbB2, and ErbB3 in the ErbB family members-coexpressing pancreatic cancer cells, which in turn results in induction of cell growth inhibition as well as apoptosis.

\section{Specific knockdown of ErbB3 expression or blocking of downstream Akt signaling enhances VPA-induced apoptosis in pancreatic cancer cells}

Upon ligand binding, ErbB family members form homodimers or heterodimers to activate the downstream signaling to exert their biological functions. Among all possible heterodimers of ErbB family members, the ErbB2/ErbB3 heterodimers have been demonstrated to be the most prevalent and potent complexes [33]. In pancreatic cancer cells, the formation of EGFR/ErbB3 heterodimers are also evidenced to influence the pancreatic cancer cells' sensitivity to erlotinib [34]. Given the fact that both the mRNA and protein expression of 
ErbB3 is comparative high in both HPAF-II and MPanc96 cells (Additional file 3: Figure S2 and Fig. 2), we then investigated whether specific down-regulation of ErbB3 expression may have any enhanced anti-survival effects in combination with VPA. While specific knockdown of ErbB3 was achieved via an ErbB3shRNA-expressing letiviral system in both HPAF-II and MPanc96 cells, downstream Akt and MAPK signalings were inhibited dramatically in parallel (Fig. 3a). The proliferation of both HPAF-II and MPanc96 cells with decreased ErbB3 expression was significantly inhibited as compared (Fig. 3b). More importantly, as compared with either ErbB3 knockdown or VPA treatment alone, the combinations of ErbB3 knockdown and VPA treatment showed significantly enhanced effects on induction of apoptosis in both HPAF-II and MPanc96 cells evidenced by increased cleavages of PARP and caspase-3 (Fig. 3c). The increased fold changes of VPA-induced apoptosis by knockdown of ErbB3 in both HPAF-II and MPanc96 cells were further verified by a specific apoptotic ELISA assay (Fig. 3d). Consistently, specific blocking of downstream Akt signaling with inhibitor of PI3K (LY294002) resulted in sensitization of HPAF-II and MPanc96 cells to VPA-induced apoptosis significantly (Fig. 3e and f). Collectively, these data suggest that specific knockdown of ErbB3 expression may enhances VPA-induced apoptosis in pancreatic cancer cells via blockade of the downstream Akt signaling.

\section{Introduced exogenous expression of either ErbB2 or ErbB3 attenuates VPA-induced inhibition of cell proliferation as well as apoptosis in pancreatic cancer cells}

Next, we sought to determine whether down-regulation of ErbB family members is required for VPA-induced growth inhibition and apoptosis in pancreatic cancer cells. Exogenous expression of either ErbB2 or ErbB3 was introduced via lentiviral expression system in HPAF-II and MPanc96 cells (Fig. 4a). Overexpression of ErbB3 significantly abrogated VPA-mediated anti-proliferation/anti-survival effects in both HPAF-II and MPanc96 cells, whereas ectopic expression of ErbB2 showed similar effects only in HPAF-II but not MPanc96 cells (Fig. 4b). Furthermore, exogenous expression of either ErbB2 or ErbB3 decreased the levels of cleaved PARP and caspase-3 induced by VPA (Fig. 4c). Significantly reduced VPA-induced apoptosis in pancreatic cancer cells with exogenous expression of either ErbB2 or ErbB3 were further demonstrated by a specific apoptotic ELISA assay (Fig. 4d). Consistently, direct activation of either ErbB3 or EGFR signaling with specific ligands (NRG-1 and EGF, respectively) also resulted in significant abrogation of anti-pancreatic cancer activity of VPA (Fig. 4e-i). Collectively, specific activation or forced overexpression of either
ErbB2 or ErbB3 attenuates VPA-induced apoptosis and inhibition of cell proliferation/survival in pancreatic cancer cells via constitutive activation of downstream Akt and MAPK signaling pathways.

\section{VPA treatment induces expression of ErbB family members-targeting microRNAs in pancreatic cancer cells without altering mRNAs levels of EGFR, ErbB2, and ErbB3} To investigate the molecular mechanism by which VPA down-regulated EGFR, ErbB2, and ErbB3 in pancreatic cancer cells, we next explored whether treatment with VPA might modulate EGFR, ErbB2, and ErbB3 mRNA levels. Conventional reverse transcriptase-PCR assay revealed that treatment with VPA did not show significant effect on the mRNA levels of EGFR, ErbB2, and ErbB3 in pancreatic cancer HPAF-II and MPanc96 cells (Fig. 5a). Similar results were further confirmed by the qRT-PCR assays (Fig. 5b). These results suggested that VPA might down-regulate EGFR, ErbB2, and ErbB3 through a transcription-independent mechanism.

Currently, miRNA emerges as an important mediator in post-transcriptional regulation of expression of gene of interest (GOI). Since we previously clarified that down-regulation of ErbB2 and ErbB3 in breast cancer cells was attributed to induction of ErbB2- and ErbB3-targeting miRNAs (miR-125a, miR-125b, and miR-205) by HDACi entinostat [24], we then asked whether similar mechanisms might account for VPA-induced down-regulation of the ErbB family members in pancreatic cancer cells. While the expression levels of miR-125a, miR-125b, and miR-205 in HPAF-II cells were markedly elevated by VPA, that of miR-125b in MPanc96 cells was also evidenced to be increased by VPA by a qRT-PCR assay (Fig. $5 \mathrm{c}$ and d). However, treatment with VPA did not alter the expression of miR-125a in MPanc96 cells, in which the expression of miR-205 was undetectable (data not shown). As both miR-133a and miR-133b had been reported to target EGFR by several studies [35-37], they were then chosen to be candidates of miRNAs targeting EGFR whose expression might be influenced by VPA. Our qRT-PCR analysis revealed that upon treatment with VPA, the expression of miR-133a was slightly increased in HPAF-II but remained unaltered in MPanc96 cells. Meanwhile, the expression of miR-133b was significantly induced in both HPAF-II and MPanc96 cells by VPA (Fig. 5d and d). Interestingly, VPA was demonstrated to specifically reduce the protein levels of endogenous but not exogenous ErbB2 and ErbB3 (Fig. 5e). Given the fact that as compared, transcripts derived from exogenous genes contain coding sequence of GOI alone but lacking of original 5'-UTR and 3'-UTR, two regions with binding sites of most miRNAs, which means that they are with less chance to be targeted by miRNAs. Taken together, 


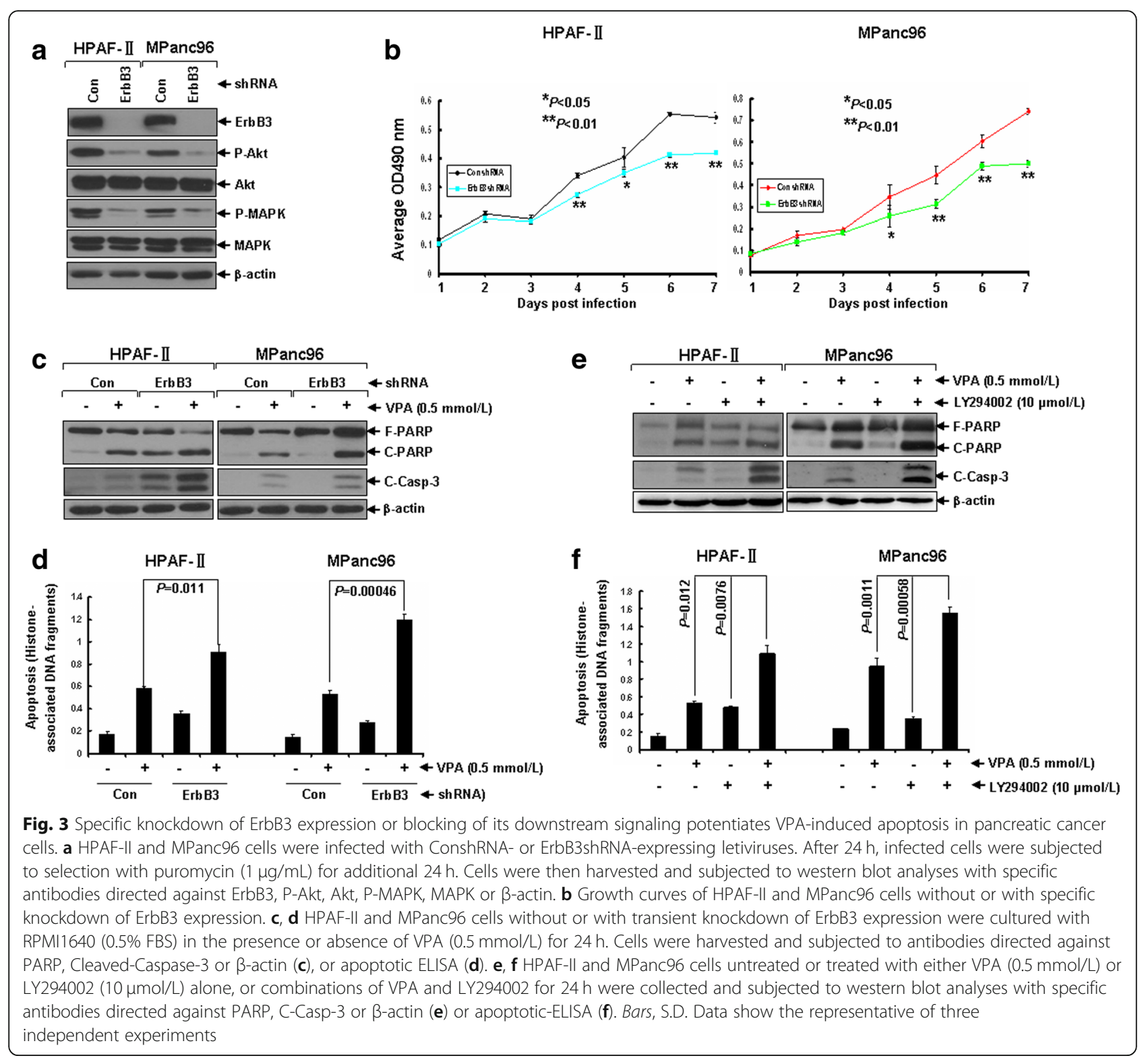

our data demonstrate that down-regulation of EGFR, ErbB2, and ErbB3 in HPAF-II and MPanc96 cells by VPA is more likely due to induction of the miRNAs that target the erbB mRNAs.

VPA inhibits tumor growth in a xenograft model of EGFR/ ErbB2/ErbB3-coexpressing pancreatic cancer

To determine whether VPA holds in vivo anti-tumor activity, we took advantage of tumor xenograft models established from HPAF-II and MPanc96 cells. When tumor volume reach $\sim 150 \mathrm{~mm}^{3}$, the tumor bearing mice were treated with either PBS or VPA $(500 \mathrm{mg} / \mathrm{kg}$ ) daily for consecutive 12 days. Our data showed that treatment with VPA significantly inhibited tumor growth as compared with PBS (Fig. 6a and b). To examine the effects of VPA on cell proliferation and apoptosis in vivo, the tumor tissues were subjected to IHC analyses of Ki-67 and cleaved caspase-3. While VPA dramatically reduced Ki-67-staining cells in both xenograft models, it significantly increased cleaved caspase- 3 only in tumor xenograft model derived from MPanc96 cells (Fig. 6c). Moreover, VPA held potential of in vivo induction of ErbB family members-targeting microRNAs and down-regulation of ErbB family members in xenograft tumors derived from both HPAF-II and MPanc96 cells (Fig. 6c, $d$ and e), which was consistent with our in vitro findings (Figs. 3 and $5 \mathrm{c}$ and d). Thus, our in vivo data further confirmed that VPA exhibits anti-tumor activity selectively against EGFR/ErbB2/ErbB3-coexpressing pancreatic cancer likely via induction of miRNAs that target the erbB mRNAs. 


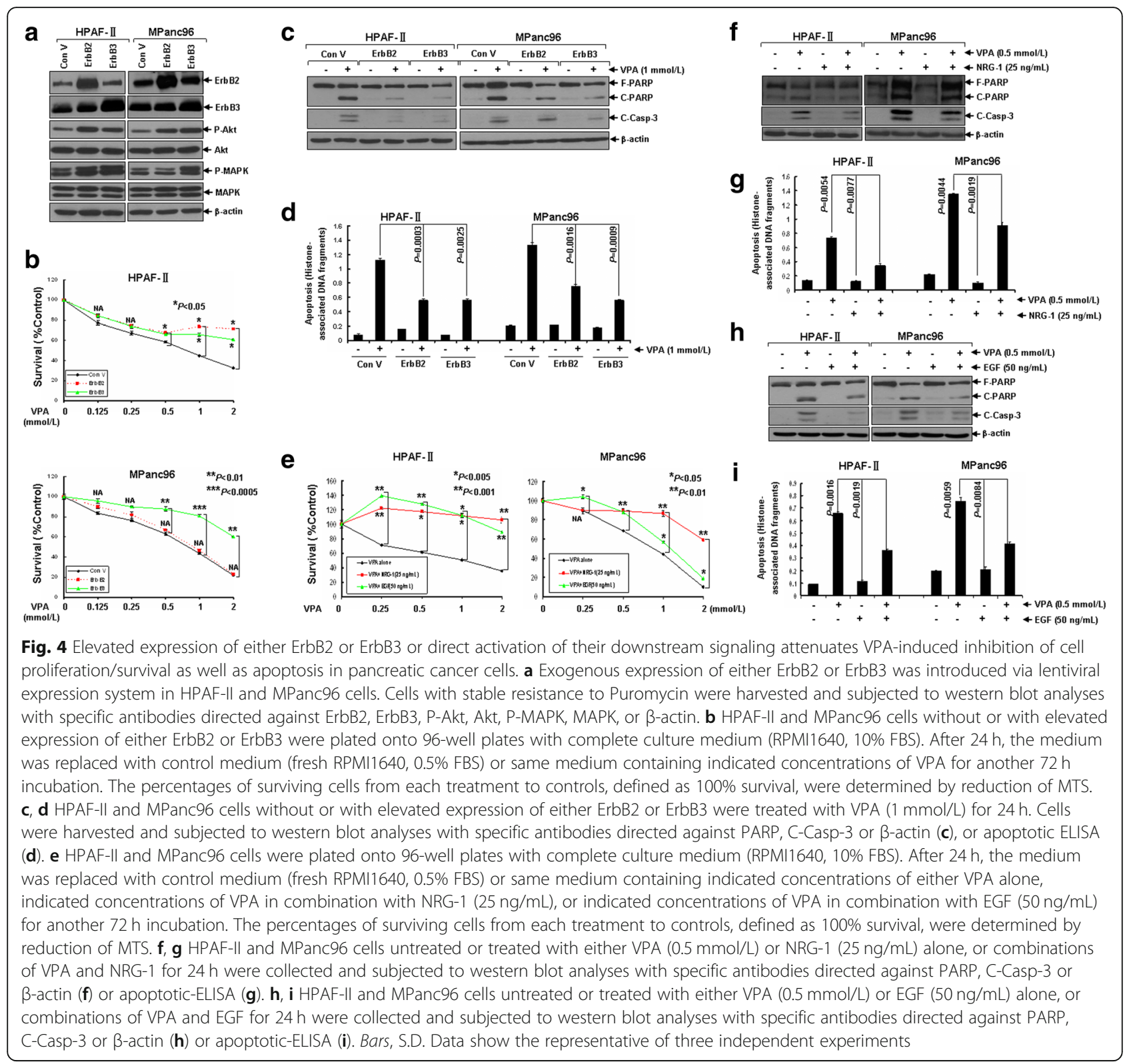

\section{Discussion}

Deregulated ErbB signalling has long been recognized as a key player in tumorigenesis of many solid tumors including pancreatic cancer [38]. Consequently, strategies of ErbB-targeting therapy have been extensively studied, among which agents directed against the EGFR are the best studied targeted agents in pancreatic cancer [9]. However, patients with pancreatic cancer benefit little from current existed therapies targeting EGFR [39, 40], which indicating that it is insufficient to overcome this disease by targeting EGFR alone. Hence, simultaneous targeting of coexpressing ErbB family members is of particular interest in developing new strategy against pancreatic cancer. In fact, several studies had showed that combined targeting of EGFR and ErbB2 did result in enhanced efficacy in treatment of pancreatic cancer $[19,20]$. In addition to EGFR and ErbB2, their kin ErbB3 is emerging as an attractive druggable target in pancreatic cancer [4143]. However, no ErbB3-targeted therapy has been approved for cancer treatment to date. In our attempt to seek novel strategy to treat pancreatic cancer, we found that VPA exhibits a promising anti-tumor activity selectively against EGFR/ErbB2/ErbB3-coexpressing pancreatic cancer. VPA not only preferentially inhibits proliferation/ survival of aforementioned subtype of pancreatic cancer cells in a dose-dependent manner (Fig. 1), but also potently induces caspase-dependent apoptosis in EGFR/ ErbB2/ErbB3-coexpressing pancreatic cancer cells within its clinically achievable range [40 100 mg/L (0.24 0.6 $\mathrm{mmol} / \mathrm{L}$ )] (Fig. 1). Mechanistically, VPA treatment was 


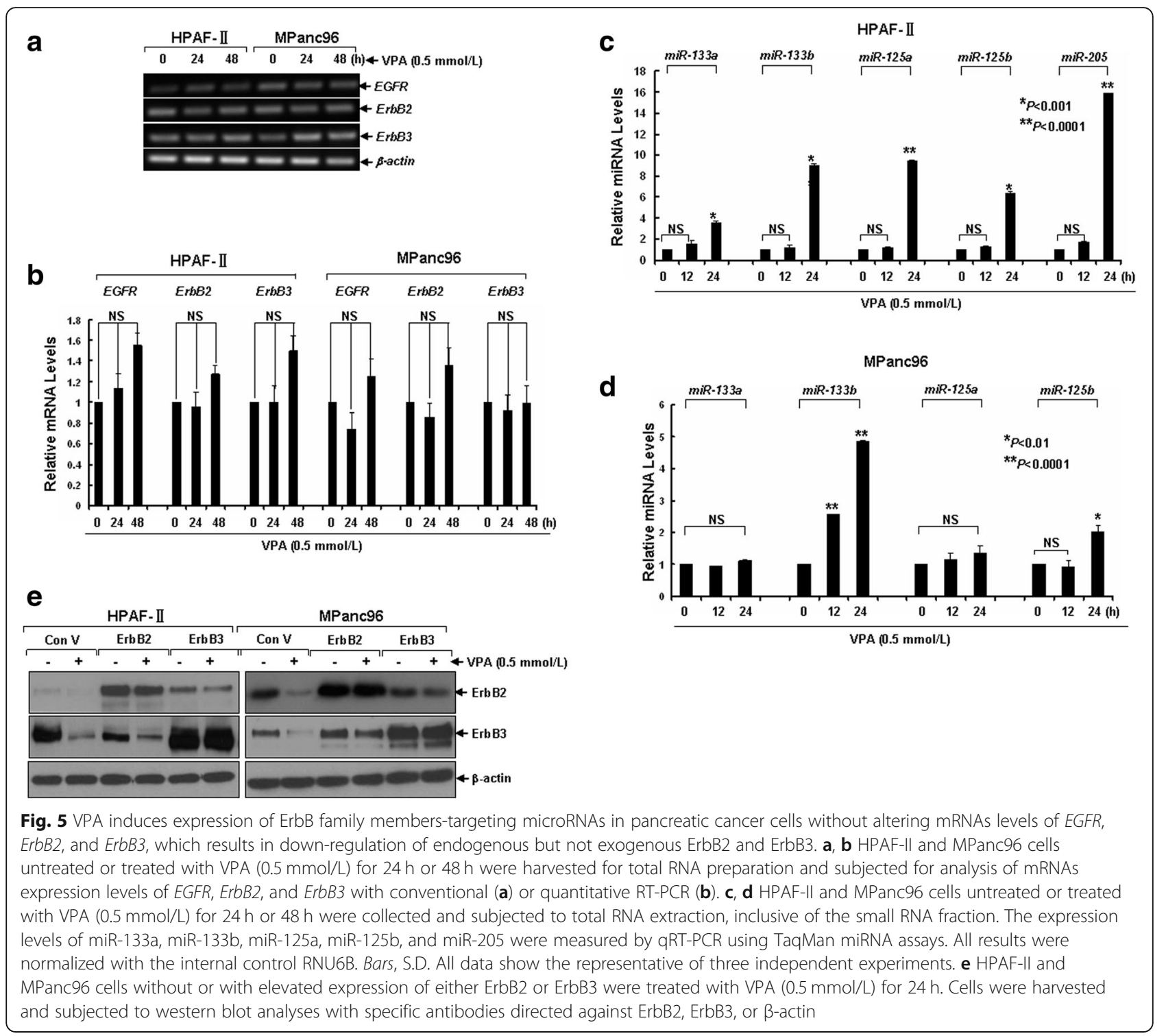

evidenced to result in significant inactivation of Akt and MAPK signalings via simultaneous down-regulation of EGFR, ErbB2, as well as ErbB3 in pancreatic cancer cells (Fig. 2). More importantly, the in vivo anti-tumor activity of VPA against ErbB family members-coexpressing pancreatic cancer was further confirmed. These findings suggest that cooperation between ErbB family members is requisite in regulating cell proliferation and survival in pancreatic cancer, thus simultaneously targeting multiple ErbB family members should be more potent than targeting individual alone. In consideration of this, VPA may be an excellent candidate drug against pancreatic cancer with a strong activity of triple-targeting EGFR, ErbB2, and ErbB3.

The anti-tumor activity of VPA has been investigated whether alone or in combination with conventional therapeutic such as epirubicin [44]. In term of molecular mechanism, VPA was showed to synergize with fluoropyrimidines in against breast and colorectal cancer via downregulating thymidylate synthase and upregulating thymidine phosphorylase [45-47]. Recently, VPA was also evidenced to synergize with capecitabine and radiotherapy in colorectal cancer via modulation of both wild-type and mutant p53 [48], and both VPA and Trichostatin A (TSA) were shown to induce pancreatic cancer cell apoptosis and autophagy by Gilardini Montani et al. [49]. Interestingly, a study by La Noce et al. indicated that VPA could induce an increased expression of stem markers including CD133, OCT4, SOX2 and NANOG in osteosarcoma cell lines, and an increased ability in sarcospheres and colonies formation efficiency, suggesting that HDAC2 is a key factor regulating both cancer stem cells (CSCs) phenotype and in vivo cancer growth [50]. We reported here that VPA-induced simultaneous 

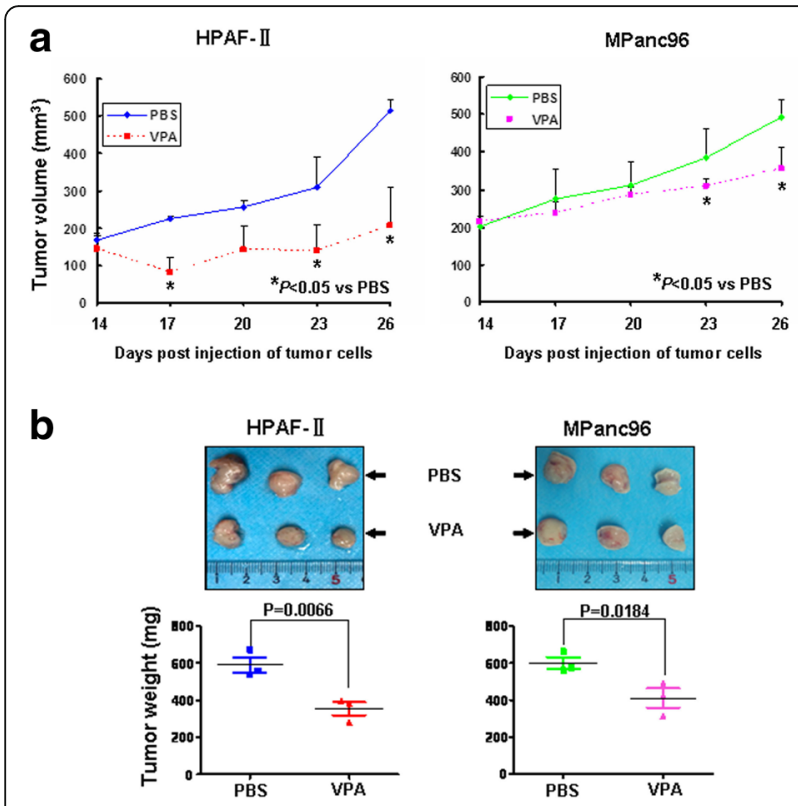

d

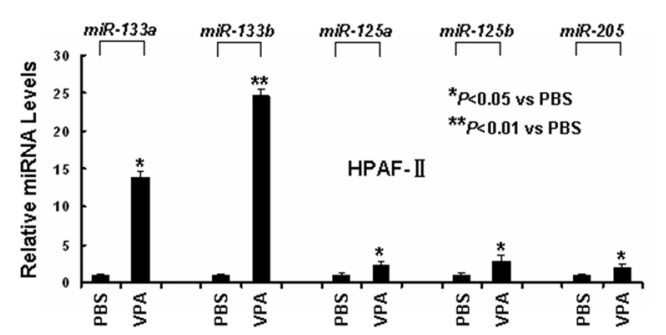

\section{C}
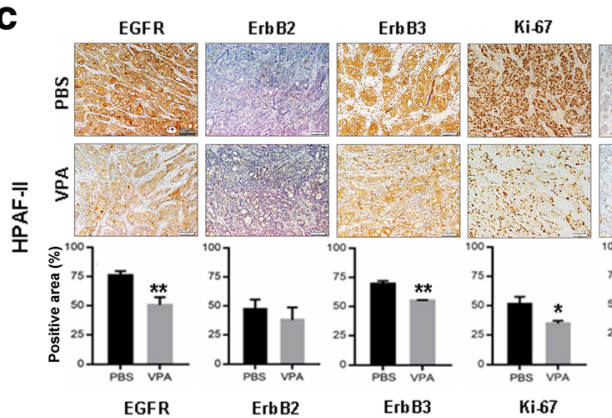

C-Casp 3

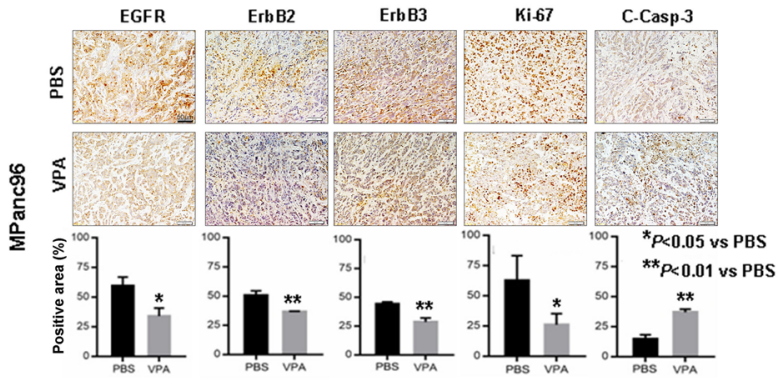

e

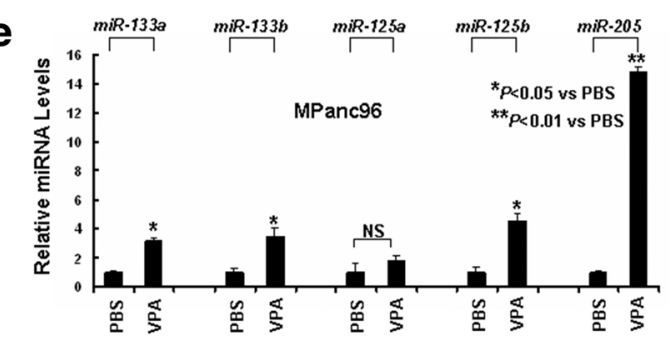

Fig. 6 VPA inhibits in vivo tumor growth of EGFR/ErbB2/ErbB3-coexpressing pancreatic cancer cells in a murine model. HPAF-II and MPanc96 cells were subcutaneously injected into nude mice to establish tumor xenografts. The tumor-bearing mice $(n=3)$ received intraperitoneal injections of either PBS or VPA (500 mg/kg) as described in the Materials and Methods. After treatments, the mice were euthanized at day 26 post injection of tumor cells and all tumors were excised for IHC and qRT-PCR analysis. a The graphs show the tumor growth curves. Bars, S.D. $\mathbf{b}$ Data show the excised tumors and tumor weights from each treatment group. c Data show the representative images of the immunostainging of EGFR, ErbB2, ErbB3, Ki-67, and C-Casp-3. The IHC slides were observed by two independent personnel. The tumor cells with positive staining of EGFR, ErbB2, ErbB3, Ki-67, or C-Casp-3 were counted from three randomly selected areas in each slide. The three areas were first identified by scanning the entire slide at $\times 100$ magnification, and then the positive-stained cells were counted at $\times 200$ magnification using an Olympus BX40 microscope. The bar graphs show the average percent of positive staining cells in each field. Bars represent S.D. ${ }^{*} P<0.05$ versus PBS control. ${ }^{*} P<0.01$ versus PBS control. d, e qRT-PCR analysis of the expression levels of miR-133a, miR-133b, miR-125a, miR-125b, and miR-205 in xenograft tumors. All results were normalized with the internal control RNU6B. Bars, S.D. All data show the representative of three independent experiments

down-regulation of EGFR, ErbB2, and ErbB3 in pancreatic cancer cells could be mainly attributed to induction of ErbB family members-targeting miRNAs both in vitro and in vivo (Figs. 5 and 6). Interestingly, upon long term treatment with VPA, silenced miR-205 was significantly induced in tumor xenograft derived from MPanc96 cells (Fig. 6e). This is similar to the mechanism underlying down-regulation of ErbB2 and ErbB3 by entinostat we found previously $[23,24]$. Nonetheless, the molecular mechanism by which VPA induces expression of ErbB family members-targeting miRNAs awaits further investigation. Our current study adds new knowledge to the understanding of mechanism of VPA against pancreatic cancer.

\section{Conclusions}

We demonstrated that VPA inhibits proliferation/survival of pancreatic cancer cells as well as induces caspase-dependent apoptosis selectively in EGFR/ErbB2/ ErbB3-coexpressing pancreatic cancer within its clinically achievable range. Mechanistic investigations revealed that VPA simultaneously down-regulates EGFR, ErbB2 and ErbB3 likely via induction of several critical miRNAs in pancreatic cancer cells, and thereby results in inactivation of downstream Akt and MAPK signaling pathways and induces cell apoptosis and growth arrest. Our data strongly suggest that VPA may be added to the treatment regimens for pancreatic cancer patients with co-overexpression of the ErbB family members. 


\section{Additional files}

Additional file 1: Table S1. Primers used for RT-PCR and qRT-PCR. (DOC $27 \mathrm{~kb}$ )

Additional file 2: Figure S1. Pro-apoptotic effect induced by VPA in EGFR/ErbB2/ErbB3-coexpressing pancreatic cancer cells. HPAF-II and MPanc96 cells untreated or treated with VPA $(0.5 \mathrm{mmol} / \mathrm{L})$ for $24 \mathrm{~h}$ or $48 \mathrm{~h}$ were harvested and subjected to flow cytometry assay. a Representative results of flow cytometry analysis. b Apoptotic effect was evaluated by flow cytometry analysis upon propidiumiodide (PI) and annexin V-FITC costaining. Data show the representative of three independent experiments. (TIF $929 \mathrm{~kb}$ )

Additional file 3: Figure S2. Relative mRNA levels of EGFR, ErbB2, and ErbB3 in pancreatic cancer cells. Parental HPAF-II and MPanc96 cells were harvested for total RNA preparation and subjected for analysis of mRNAs expression levels of EGFR, ErbB2, and ErbB3 with quantitative RT-PCR. Bars, S.D. Data show the representative of three independent experiments. (TIF $121 \mathrm{~kb})$

\section{Abbreviations}

EGFR: Epidermal growth factor receptor; ELISA: Enzyme-linked immunosorbent assay; ErbB: Erythroblastic leukemia viral oncogene homolog; GOI: Gene of interest; HDAC: Histone deacetylase; HDACi: HDAC inhibitor; IHC: Immunohistochemistry; MAPK: Mitogen-activated protein kinase; MTS: 3-(4,5-dimethylthiazol-2-yl)-5-(3-carboxymethoxyphenyl)-2-(4sulfophenyl)-2H-tetrazolium, inner salt; PARP: Poly (ADP-ribose) polymerase PI-3 K: Phosphoinositide 3-kinase; qRT-PCR: Real-time quantitative reverse transcriptase PCR; RTKs: Receptor tyrosine kinases; UTR: Untranslated region; VPA: Valproic acid

\section{Acknowledgements}

Not applicable

\section{Funding}

This study was supported in part by the National Natural Science Foundation of China No.81772848, Joint Funds for the Innovation of Science and Technology from Fujian Province No.2017Y9127, and the Natural Science Foundation of Fujian Province of China No.2016 J01577 to S, Wang. This study was also partially supported by the National Natural Science Foundation of China No.81572452 to Y, Peng and No.81703225 to P, Wang.

\section{Availability of data and materials}

All data generated or analyzed during this research are included in this manuscript.

\section{Authors' contributions}

The authors' contributions to this research work are reflected in the order shown. TL, QR, and WZ carried out the majority of experiments. RJ, LX and $\mathrm{RL}$ performed cell culture. $\mathrm{HZ}, \mathrm{JC}$ and $\mathrm{YL}$ participated in performing western blotting analysis. PW and HD prepared lentiviruses. $\mathrm{LH}$ and JC helped in doing QRT-PCR analysis. YP and ZY carried out animal experiments. TM and SW conceived of the study, supervised the research and finalized the report. All authors read and approved the final manuscript.

\section{Ethics approval and consent to participate}

This study was approved by the Ethics Committee of Affiliated Dongfang Hospital of Xiamen University School of Medicine. Experiments with animals were performed in accordance to the guidelines for experimental animal managements established by Xiamen University.

\section{Consent for publication}

Not applicable.

\section{Competing interests}

The authors declare that they have no competing interests.

\section{Publisher's Note}

Springer Nature remains neutral with regard to jurisdictional claims in published maps and institutional affiliations.

\section{Author details}

${ }^{1}$ Department of Urology, The 900th Hospital of the Joint Logistics Team (the Former Fuzhou General Hospital), Fujian Medical University, Fuzhou 350025, China. 156 Xi'er Huan Bei Road, Fuzhou 350025, Fujian Province, China. ${ }^{2}$ Fujian Key Laboratory of Transplant Biology, Affiliated Dongfang Hospital, Xiamen University School of Medicine, Fuzhou 350025, Fujian Province, China. ${ }^{3}$ Department of Medical Oncology, First Hospital of Sanming, Sanming 365000, Fujian Province, China. ${ }^{4}$ Department of Medical Ultrasound, Guangzhou First Peoples's Hospital, Guangzhou Medical University, Guangdong Province, Guangzhou 510180, China. ${ }^{5}$ Department of Clinical Medicine, Fujian Health Vocational and Technical College, Fuzhou 350101, Fujian Province, China. ${ }^{6}$ Department of Medical Oncology, The 900th Hospital of the Joint Logistics Team (the Former Fuzhou General Hospital), Fujian Medical University, Fuzhou 350025, Fujian Province, China.

Received: 5 February 2019 Accepted: 31 March 2019

Published online: 08 April 2019

\section{References}

1. Torre LA, Bray F, Siegel RL, Ferlay J, Lortet-Tieulent J, Jemal A. Global cancer statistics, 2012. CA Cancer J Clin. 2015;65:87-108.

2. Hidalgo M. Pancreatic cancer. N Engl J Med. 2010;362:1605-17.

3. Li D, Xie K, Wolff R, Abbruzzese JL. Pancreatic cancer. Lancet. 2004;363: 1049-57.

4. Siegel RL, Miller KD, Fedewa SA, Ahnen DJ, Meester RGS, Barzi A, Jemal A. Colorectal cancer statistics, 2017. CA Cancer J Clin. 2017;67:177-93.

5. Bardeesy N, DePinho RA. Pancreatic cancer biology and genetics. Nat Rev Cancer. 2002;2:897-909.

6. Habbe N, Shi G, Meguid RA, Fendrich V, Esni F, Chen H, et al. Spontaneous induction of murine pancreatic intraepithelial neoplasia (mPanIN) by acinar cell targeting of oncogenic Kras in adult mice. Proc Natl Acad Sci U S A. 2008;105:18913-8.

7. De La OJ, Emerson LL, Goodman JL, Froebe SC, Illum BE, Curtis AB, et al. Notch and Kras reprogram pancreatic acinar cells to ductal intraepithelial neoplasia. Proc Natl Acad Sci U S A. 2008;105:18907-12.

8. Makohon-Moore A, lacobuzio-Donahue CA. Pancreatic cancer biology and genetics from an evolutionary perspective. Nat Rev Cancer. 2016;16:553-65.

9. Wolfgang $C L$, Herman JM, Laheru DA, Klein AP, Erdek MA, Fishman EK, et al. Recent progress in pancreatic cancer. CA Cancer J Clin. 2013;63:318-48.

10. Waddell N, Pajic M, Patch AM, Chang DK, Kassahn KS, Bailey P, et al. Whole genomes redefine the mutational landscape of pancreatic cancer. Nature. 2015;518:495-501.

11. Biankin AV, Waddell N, Kassahn KS, Gingras MC, Muthuswamy LB, Johns AL, et al. Pancreatic cancer genomes reveal aberrations in axon guidance pathway genes. Nature. 2012;491:399-405.

12. Yachida S, White CM, Naito Y, Zhong Y, Brosnan JA, Macgregor-Das AM, et al. Clinical significance of the genetic landscape of pancreatic cancer and implications for identification of potential long-term survivors. Clin Cancer Res. 2012;18:6339-47.

13. Pylayeva-Gupta Y, Grabocka E, Bar-Sagi D. RAS oncogenes: weaving a tumorigenic web. Nat Rev Cancer. 2011;11:761-74.

14. Lemmon MA, Schlessinger J. Cell signaling by receptor tyrosine kinases. Cell. 2010;141:1117-34.

15. Arteaga $\mathrm{CL}$, Engelman JA. ERBB receptors: from oncogene discovery to basic science to mechanism-based cancer therapeutics. Cancer Cell. 2014; 25:282-303.

16. Friess H, Wang L, Zhu Z, Gerber R, Schroder M, Fukuda A, et al. Growth factor receptors are differentially expressed in cancers of the papilla of vater and pancreas. Ann Surg. 1999;230:767-74.

17. Thomas G, Chardes T, Gaborit N, Mollevi C, Leconet W, Robert B, et al. HER3 as biomarker and therapeutic target in pancreatic cancer: new insights in pertuzumab therapy in preclinical models. Oncotarget. 2014;5:7138-48.

18. Larbouret C, Robert B, Navarro-Teulon I, Thezenas S, Ladjemi MZ, Morisseau $\mathrm{S}$, et al. In vivo therapeutic synergism of anti-epidermal growth factor receptor and anti-HER2 monoclonal antibodies against pancreatic carcinomas. Clin Cancer Res. 2007;13:3356-62.

19. Maron R, Schechter B, Mancini M, Mahlknecht G, Yarden Y, Sela M. Inhibition of pancreatic carcinoma by homo- and heterocombinations of antibodies against EGF-receptor and its kin HER2/ErbB-2. Proc Natl Acad Sci U S A. 2013;110:15389-94. 
20. Gore J, Imasuen-Williams IE, Conteh AM, Craven KE, Cheng M, Korc M. Combined targeting of TGF- $\beta$, EGFR and HER2 suppresses lymphangiogenesis and metastasis in a pancreatic cancer model. Cancer Lett. 2016:379:143-53.

21. Bolden JE, Peart MJ, Johnstone RW. Anticancer activities of histone deacetylase inhibitors. Nat Rev Drug Discov. 2006;5:769-84.

22. Minucci S, Pelicci PG. Histone deacetylase inhibitors and the promise of epigenetic (and more) treatments for cancer. Nat Rev Cancer. 2006;6:38-51.

23. Huang X, Gao L, Wang S, Lee CK, Ordentlich P, Liu B. HDAC inhibitor SNDX275 induces apoptosis in erbB2-overexpressing breast cancer cells via down-regulation of erbB3 expression. Cancer Res. 2009;69:8403-11.

24. Wang S, Huang J, Lyu H, Lee CK, Tan J, Wang J, et al. Functional cooperation of miR-125a, miR-125b, and miR-205 in entinostat-induced downregulation of erbB2/erbB3 and apoptosis in breast cancer cells. Cell Death Dis. 2013;4:e556.

25. Botrugno OA, Robert T, Vanoli F, Foiani M, Minucci S. Molecular pathways: old drugs define new pathways: non-histone acetylation at the crossroads of the DNA damage response and autophagy. Clin Cancer Res. 2012;18: 2436-42.

26. Lee CK, Wang S, Huang X, Ryder J, Liu B. HDAC inhibition synergistically enhances alkylator-induced DNA damage responses and apoptosis in multiple myeloma cells. Cancer Lett. 2010;296:233-40.

27. Wang S, Huang X, Lee CK, Liu B. Elevated expression of erbB3 confers paclitaxel resistance in erbB2-overexpressing breast cancer cells via upregulation of Survivin. Oncogene. 2010;29:4225-36.

28. Wang S, Zhu L, Zuo W, Zeng Z, Huang L, Lin F, et al. MicroRNA-mediated epigenetic targeting of Survivin significantly enhances the antitumor activity of paclitaxel against non-small cell lung cancer. Oncotarget. 2016;7:37693-713.

29. Gui CY, Ngo L, Xu WS, Richon VM, Marks PA. Histone deacetylase (HDAC) inhibitor activation of p21WAF1 involves changes in promoter-associated proteins, including HDAC1. Proc Natl Acad Sci U S A. 2004;101:1241-6.

30. Hanahan D, Weinberg RA. Hallmarks of cancer: the next generation. Cell. 2011;144:646-74.

31. Chandarlapaty S, Sawai A, Scaltriti M, Rodrik-Outmezguine V, Grbovic-Huezo $\mathrm{O}$, Serra $\mathrm{V}$, et al. AKT inhibition relieves feedback suppression of receptor tyrosine kinase expression and activity. Cancer Cell. 2011;19:58-71.

32. Chakrabarty A, Sanchez V, Kuba MG, Rinehart C, Arteaga CL. Feedback upregulation of HER3 (ErbB3) expression and activity attenuates antitumor effect of PI3K inhibitors. Proc Natl Acad Sci U S A. 2012;109:2718-23.

33. Citri A, Skaria KB, Yarden $Y$. The deaf and the dumb: the biology of ErbB-2 and ErbB-3. Exp Cell Res. 2003;284:54-65.

34. Frolov A, Schuller K, Tzeng CW, Cannon EE, Ku BC, Howard JH, et al. ErbB3 expression and dimerization with EGFR influence pancreatic cancer cell sensitivity to erlotinib. Cancer Biol Ther. 2007;6:548-54.

35. Wei W, Liu Y, Lu Y, Yang B, Tang L. LncRNA XIST promotes pancreatic Cancer proliferation through miR-133a/EGFR. J Cell Biochem. 2017;118:3349-58.

36. Tang Y, Pan J, Huang S, Peng X, Zou X, Luo Y, et al. Downregulation of miR$133 a-3 p$ promotes prostate cancer bone metastasis via activating PI3K signaling. J Exp Clin Cancer Res. 2018;37:160

37. Liu L, Shao X, Gao W, Zhang Z, Liu P, Wang R, et al. MicroRNA-133b inhibits the growth of non-small-cell lung cancer by targeting the epidermal growth factor receptor. FEBS J. 2012;279:3800-12.

38. Citri A, Yarden Y. EGF-ERBB signalling: towards the systems level. Nat Rev Mol Cell Biol. 2006;7:505-16.

39. Crane CH, Varadhachary GR, Yordy JS, Staerkel GA, Javle MM, Safran H, et al. Phase II trial of cetuximab, gemcitabine, and oxaliplatin followed by chemoradiation with cetuximab for locally advanced (T4) pancreatic adenocarcinoma: correlation of Smad4 (Dpc4) immunostaining with pattern of disease progression. J Clin Oncol. 2011;29:3037-43.

40. Moore MJ, Goldstein D, Hamm J, Figer A, Hecht JR, Gallinger S, et al. Erlotinib plus gemcitabine compared with gemcitabine alone in patients with advanced pancreatic Cancer: a phase III trial of the National Cancer Institute of Canada clinical trials group. J Clin Oncol. 2007;25:1960-6.

41. Lakshmanan I, Seshacharyulu P, Haridas D, Rachagani S, Gupta S, Joshi S, et al. Novel HER3/MUC4 oncogenic signaling aggravates the tumorigenic phenotypes of pancreatic cancer cells. Oncotarget. 2015;6:21085-99.

42. Heining $\mathrm{C}$, Horak $\mathrm{P}$, Uhrig $\mathrm{S}$, Codo PL, Klink B, Hutter $\mathrm{B}$, et al. NRG1 fusions in KRAS wild-type pancreatic Cancer. Cancer Discov. 2018;8:1087-95.

43. Ogier C, Colombo PE, Bousquet C, Canterel-Thouennon L, Sicard P, Garambois V, et al. Targeting the NRG1/HER3 pathway in tumor cells and cancer-associated fibroblasts with an anti-neuregulin 1 antibody inhibits tumor growth in pre-clinical models of pancreatic cancer. Cancer Lett. 2018, 432:227-36.

44. Marchion DC, Bicaku E, Daud Al, Sullivan DM, Munster PN. In vivo synergy between topoisomerase II and histone deacetylase inhibitors: predictive correlates. Mol Cancer Ther. 2005;4:1993-2000.

45. Di Gennaro E, Bruzzese F, Pepe S, Leone A, Delrio P, Subbarayan PR, et al. Modulation of thymidilate synthase and p53 expression by HDAC inhibitor vorinostat resulted in synergistic antitumor effect in combination with 5FU or raltitrexed. Cancer Biol Ther. 2009;8:782-91.

46. Di Gennaro E, Piro G, Chianese Ml, Franco R, Di Cintio A, Moccia T, et al. Vorinostat synergises with capecitabine through upregulation of thymidine phosphorylase. Br J Cancer. 2010;103:1680-91.

47. Terranova-Barberio M, Roca MS, Zotti Al, Leone A, Bruzzese F, Vitagliano C, et al. Valproic acid potentiates the anticancer activity of capecitabine in vitro and in vivo in breast cancer models via induction of thymidine phosphorylase expression. Oncotarget. 2016;7:7715-31.

48. Terranova-Barberio M, Pecori B, Roca MS, Imbimbo S, Bruzzese F, Leone A, et al. Synergistic antitumor interaction between valproic acid, capecitabine and radiotherapy in colorectal cancer: critical role of p53. J Exp Clin Cancer Res. 2017;36:177.

49. Gilardini Montani MS, Granato M, Santoni C, Del Porto P, Merendino N, D'Orazi G, et al. Histone deacetylase inhibitors VPA and TSA induce apoptosis and autophagy in pancreatic cancer cells. Cell Oncol (Dordr). 2017:40:167-80.

50. La Noce M, Paino F, Mele L, Papaccio G, Regad T, Lombardi A, et al. HDAC2 depletion promotes osteosarcoma's stemness both in vitro and in vivo: a study on a putative new target for CSCs directed therapy. J Exp Clin Cancer Res. 2018;37:296.

\section{Ready to submit your research? Choose BMC and benefit from:}

- fast, convenient online submission

- thorough peer review by experienced researchers in your field

- rapid publication on acceptance

- support for research data, including large and complex data types

- gold Open Access which fosters wider collaboration and increased citations

- maximum visibility for your research: over $100 \mathrm{M}$ website views per year

At $\mathrm{BMC}$, research is always in progress.

Learn more biomedcentral.com/submissions 